\title{
A new calculation technique for the Laplace and Sumudu transforms by means of the variational iteration method
}

\author{
Murat Gubes ${ }^{1}$
}

Received: 10 January 2018 / Accepted: 26 November 2018 / Published online: 3 December 2018

(c) The Author(s) 2018

\begin{abstract}
The aim of this study is to calculate the well-known Laplace and Sumudu transforms of functions in a different way. For our purpose, we present a computational tool by applying the variational iteration method. The Laplace and Sumudu transforms of some of the basic functions are also given as illustrations to test the efficiency and reliability of the proposed computational method.
\end{abstract}

Keywords Laplace transform · Sumudu transform · Variational iteration method · Linear IVP

Mathematics Subject Classification 00A69 -34A25 · 44A10 · 65R10

\section{Introduction}

The variational iteration method (VIM) is one of the powerful mathematical tools to solve various kinds of linear and nonlinear problems which was proposed by He [1-3]. Besides these, variational iteration method and its modifications are also used in many areas of mathematics and science as seen [4-7] and many others. In recent times, Fatoorehchi et al. [8] have performed the differential transform method (DTM) to obtain Laplace transform of functions. Also, the applications of homotopy perturbation (HPM) and adomian decomposition (ADM) methods for calculating Laplace transform are seen in the literature $[9,10]$, respectively.

In that respect, we aim to give a new calculation of Laplace and Sumudu transforms using the VIM which will be the first time in the literature. Therefore, we take into account the two elementary first-order linear ODEs as follow

$$
\begin{aligned}
& \theta(t)^{\prime}=p \theta(t)+Q(t) \\
& \theta(0)=0
\end{aligned}
$$

and

Murat Gubes

mrtgbs@gmail.com

1 Department of Mathematics, Science Faculty of Selcuk University, Konya, Turkey

$$
f(t)^{\prime}=\frac{1}{r} f(t)+\frac{1}{r} Q(t)
$$$$
f(0)=0
$$

where $p$ and $r$ are positive constants. We can easily obtain the analytical solutions of (1) and (2) as below

$\theta(t) \mathrm{e}^{-p t}=\int\left(Q(t) \mathrm{e}^{-p t}\right) \mathrm{d} t$

and

$f(t) \mathrm{e}^{-\frac{t}{r}}=\frac{1}{r} \int\left(Q(t) \mathrm{e}^{-\frac{t}{r}}\right) \mathrm{d} t$

It is pointed out that if the right-hand side of Eqs. (3) and (4) is considered as definite integration from zero to infinity, then we obtain the Laplace and Sumudu transforms of the functions $Q(t)$ from right-hand side of the equations as

$G(p)=L[Q(t)]=\int_{0}^{\infty}\left(Q(t) \mathrm{e}^{-p t}\right) \mathrm{d} t=\left[\theta(t) \mathrm{e}^{-p t}\right]_{t=0}^{t=\infty}$

$$
\begin{aligned}
H(r) & =S[Q(t)]=\int_{0}^{\infty}\left(Q(r t) \mathrm{e}^{-t}\right) \mathrm{d} t \\
& =\frac{1}{r} \int_{0}^{\infty}\left(Q(t) \mathrm{e}^{-\frac{t}{r}}\right) \mathrm{d} t=\left[f(t) \mathrm{e}^{-\frac{t}{r}}\right]_{t=0}^{t=\infty}
\end{aligned}
$$


where $G(p)$ and $H(r)$ are the Laplace and Sumudu transform of $Q(t)$ functions, respectively. Also, $L$ and $S$ denote the Laplace and Sumudu operator.

\section{Implementation of VIM to solve (1) and (2)}

In order to make a basic definition of the VIM, we consider the following general nonlinear problem

$V[u(t)]+N[u(t)]=g(t)$

where $V$ is a linear operator, $N$ is a nonlinear operator and $g$ is a given continuous function. According to the original VIM, we construct the correction functional as [1-3]

$u_{m+1}(t)=u_{m}(t)+\int_{0}^{t} \lambda(\tau)\left[V\left(u_{m}(\tau)\right)+N\left(\tilde{u}_{m}(\tau)\right)-g(\tau)\right] \mathrm{d} \tau, \quad m \geq 0$

Here $\lambda$ is a Lagrange multiplier obtained by the variational theory [1-3], $\tilde{u}_{m}$ is considered as a restricted variation [1-3], i.e. $\delta \tilde{u}_{m}=0$. Firstly, we determine the Lagrange multiplier which can be identified optimally via the variational theory [1-3]. Then, successive iterations $u_{m}(t), m \geq 0$ are obtained by using Lagrange multiplier and initial approximate function $u_{0}$ that satisfies, at least, the initial and boundary conditions with possible unknowns. Consequently, the exact solution of (7) can be obtained by the following formula

$u(t)=\lim _{m \rightarrow \infty} u_{m}(t)$

Now, we perform the VIM to solve (1) and (2). By considering the initial conditions, we can write $\theta_{0}(t)=0$ and $f_{0}(t)=0$. If we apply the VIM procedure (7), (8) to (1) and (2), then Lagrange multipliers $\lambda_{\theta}(\tau)$ and $\lambda_{f}(\tau)$ are obtained as below

$\lambda_{\theta}(\tau)=-\mathrm{e}^{p(t-\tau)}, \quad \lambda_{f}(\tau)=-\mathrm{e}^{\frac{1}{r}(t-\tau)}$

Therefore, considering (8), (9) and (10), we obtain the iteration formula for the VIM solutions of (1) and (2) as follow, respectively,

$\theta_{m+1}(t)=\theta_{m}(t)-\int_{0}^{t}\left(\mathrm{e}^{p(t-\tau)}\left[\theta_{m}^{\prime}(\tau)-p \theta_{m}(\tau)-Q(\tau)\right]\right) \mathrm{d} \tau$

$f_{m+1}(t)=f_{m}(t)-\int_{0}^{t}\left(\mathrm{e}^{\frac{1}{r}(t-\tau)}\left[f_{m}^{\prime}(\tau)-\frac{1}{r} f_{m}(\tau)-\frac{1}{r} Q(\tau)\right]\right) \mathrm{d} \tau$

Here $m \geq 0$. By substituting the first iteration of (11) and (12) to right-hand side of (5) and (6), we obtain the Laplace and Sumudu transform of $Q(t)$ functions by means of the VIM. Namely,
$L[Q(t)]=G(p)=\left[\mathrm{e}^{-p t} \theta_{1}(t)\right]_{t=0}^{t=\infty}$

$S[Q(t)]=H(r)=\left[\mathrm{e}^{-\frac{t}{r}} f_{1}(t)\right]_{t=0}^{t=\infty}$

\section{Computing the Laplace and Sumudu transforms via VIM}

In this section, Laplace and Sumudu transforms of some of the frequently used functions, especially used in the applied sciences, are obtained to show the efficiency and accuracy of the proposed computational method.

\section{For Laplace transform}

Case 1 Let $Q(t)=t^{n}, n \geq 0$. By considering (1), (5) and (13), it can be written as

$L\left[t^{n}\right]=\left[\mathrm{e}^{-p t} \theta_{1}(t)\right]_{t=0}^{t=\infty}$

where $\theta_{1}(t)$ is obtained from (11) as

$\theta_{1}(t)=\frac{\mathrm{e}^{p t}}{p^{n+1}}[\Gamma(n+1)-\Gamma(n+1, p t)]$

where $\Gamma(n), \Gamma(n, t)$ are the well-known gamma and incomplete gamma functions, respectively. Thus, we have the Laplace transform of $Q(t)=t^{n}$ as

$$
\begin{aligned}
L\left[t^{n}\right] & =\left[\mathrm{e}^{-p t}\left(\frac{\mathrm{e}^{p t}}{p^{n+1}}[\Gamma(n+1)-\Gamma(n+1, p t)]\right)\right]_{t=0}^{t=\infty} \\
& =\frac{1}{p^{n+1}}[(n !-0)-(n !-n !)]=\frac{n !}{p^{n+1}}
\end{aligned}
$$

Case 2 Let $Q(t)=\cosh (a t), p>a$. From (11), we obtain $\theta_{1}(t)$ as following

$\theta_{1}(t)=\frac{p \mathrm{e}^{p t}}{p^{2}-a^{2}}-\frac{p\left(\mathrm{e}^{a t}+\mathrm{e}^{-a t}\right)}{2\left(p^{2}-a^{2}\right)}-\frac{a\left(\mathrm{e}^{a t}-\mathrm{e}^{-a t}\right)}{2\left(p^{2}-a^{2}\right)}$

Using the (1), (5), (13) and (18), we get the Laplace transform of $Q(t)=\cosh (a t)$

$$
\begin{aligned}
L[\cosh (a t)]= & \left\{\frac{p}{p^{2}-a^{2}}-\frac{p\left(\mathrm{e}^{(a-p) t}+\mathrm{e}^{-(a+p) t}\right)}{2\left(p^{2}-a^{2}\right)}\right. \\
& \left.-\frac{a\left(\mathrm{e}^{(a-p) t}-\mathrm{e}^{-(a+p) t}\right)}{2\left(p^{2}-a^{2}\right)}\right\}_{t=0}^{t=\infty} \\
= & \frac{p}{p^{2}-a^{2}}-0-\frac{p}{p^{2}-a^{2}}+\frac{p}{p^{2}-a^{2}} \\
= & \frac{p}{p^{2}-a^{2}}
\end{aligned}
$$


Case 3 Let $Q(t)=\mathrm{e}^{a t}, p>a$. We have the $\theta_{1}(t)$ as

$\theta_{1}(t)=\frac{\mathrm{e}^{p t}-\mathrm{e}^{a t}}{p-a}$

By substituting (20) into the right-hand side of (13), the Laplace transform of $Q(t)=\mathrm{e}^{a t}$ is obtained as

$$
\begin{aligned}
L\left[\mathrm{e}^{a t}\right] & =\left\{\frac{1-\mathrm{e}^{(a-p) t}}{p-a}\right\}_{t=0}^{t=\infty} \\
& =\frac{1-0}{p-a}-\frac{1-1}{p-a}=\frac{1}{p-a}
\end{aligned}
$$

Case 4 Let $Q(t)=t \cos (a t)$. From (11), we have the $\theta_{1}(t)$ as

$$
\begin{aligned}
\theta_{1}(t)= & \frac{\left(p^{2}-a^{2}\right) \mathrm{e}^{p t}}{\left(p^{2}+a^{2}\right)^{2}} \\
& -\frac{\left[t p^{3}+t p a^{2}+p^{2}-a^{2}\right] \cos (a t)}{\left(p^{2}+a^{2}\right)^{2}} \\
& +\frac{\left[t a p^{2}+t a^{3}+2 a p\right] \sin (a t)}{\left(p^{2}+a^{2}\right)^{2}}
\end{aligned}
$$

and put (22) into right-hand side of (13), then we obtain the Laplace transform of $Q(t)=t \cos (a t)$ as following

$$
\begin{aligned}
L[t \cos (a t)]= & \left\{\frac{p^{2}-a^{2}}{\left(p^{2}+a^{2}\right)^{2}}-\frac{\left[t p^{3}+t p a^{2}+p^{2}-a^{2}\right] \cos (a t)}{\mathrm{e}^{p t}\left(p^{2}+a^{2}\right)^{2}}\right. \\
& \left.+\frac{\left[t a p^{2}+t a^{3}+2 a p\right] \sin (a t)}{\mathrm{e}^{p t}\left(p^{2}+a^{2}\right)^{2}}\right\}_{t=0}^{t=\infty} \\
= & \frac{p^{2}-a^{2}}{\left(p^{2}+a^{2}\right)^{2}}-0+0-0-\left(\frac{p^{2}-a^{2}}{\left(p^{2}+a^{2}\right)^{2}}-\frac{p^{2}-a^{2}}{\left(p^{2}+a^{2}\right)^{2}}\right) \\
= & \frac{p^{2}-a^{2}}{\left(p^{2}+a^{2}\right)^{2}}
\end{aligned}
$$

Case 5 Let $Q(t)=\operatorname{erf}(t)$. By considering (1), (5), (11) and (13), we can easily obtain

$\theta_{1}(t)=-\frac{\mathrm{e}^{p t+\frac{p^{2}}{4}} \operatorname{erf}\left(\frac{p}{2}\right)+\operatorname{erf}(t)}{p}+\frac{\mathrm{e}^{p t+\frac{p^{2}}{4}} \operatorname{erf}\left(t+\frac{p}{2}\right)}{p}$

and

$$
\begin{aligned}
L[\operatorname{erf}(t)] & =\left\{-\frac{\mathrm{e}^{\frac{p^{2}}{4}} \operatorname{erf}\left(\frac{p}{2}\right)+\mathrm{e}^{-p t} \operatorname{erf}(t)}{p}+\frac{\mathrm{e}^{\frac{p^{2}}{4}} \operatorname{erf}\left(t+\frac{p}{2}\right)}{p}\right\}_{t=0}^{t=\infty} \\
& =\frac{-\mathrm{e}^{\frac{p^{2}}{4}} \operatorname{erf}\left(\frac{p}{2}\right)-0+\mathrm{e}^{\frac{p^{2}}{4}}}{p}+\frac{\mathrm{e}^{\frac{p^{2}}{4}} \operatorname{erf}\left(\frac{p}{2}\right)+0-\mathrm{e}^{\frac{p^{2}}{4}} \operatorname{erf}\left(\frac{p}{2}\right)}{p} \\
& =\frac{\mathrm{e}^{\frac{p^{2}}{4}}\left(1-\operatorname{erf}\left(\frac{p}{2}\right)\right)}{p}=\frac{\mathrm{e}^{\frac{p^{2}}{4}} \operatorname{erfc}\left(\frac{p}{2}\right)}{p}
\end{aligned}
$$

Here $\operatorname{erf}(t)$ and $\operatorname{erfc}(t)$ are well-known error and complementary error functions, respectively.

Case 6 Let $Q(t)=\ln (t)$. Once again using the (11), we obtain $\theta_{1}(t)$ as

$\theta_{1}(t)=-\frac{\ln (t)+\mathrm{e}^{p t} E_{1}(p t)}{p}-\frac{\mathrm{e}^{p t} \gamma+\mathrm{e}^{p t} \ln (p)}{p}$

where $\gamma=0.5772156649 \ldots$ is the Eulers constant and $E_{1}(t)$ is a well-known exponential integral defined as

$E_{1}(t)=\int_{t}^{\infty} \frac{\mathrm{e}^{-v}}{v} \mathrm{~d} v$

If the same process is applied as in before, we get

$$
\begin{aligned}
L[\ln (t)] & =\left\{\frac{-\mathrm{e}^{-p t} \ln (t)-E_{1}(p t)}{p}-\frac{\gamma+\ln (p)}{p}\right\}_{t=0}^{t=\infty} \\
& =\frac{-0-0-\gamma-\ln (p)}{p}+\frac{\ln (t)-\gamma-\ln (p t)+\gamma+\ln (p)}{p} \\
& =\frac{-\gamma-\ln (p)}{p}
\end{aligned}
$$

\section{For Sumudu transform}

Case 1 Let $Q(t)=t^{n}, n \geq 0$. By using (2), (6) and (14), it can be written as

$S\left[t^{n}\right]=\left[\mathrm{e}^{-\frac{t}{r}} f_{1}(t)\right]_{t=0}^{t=\infty}$

where $f_{1}(t)$ is obtained from (12) as

$f_{1}(t)=t^{n} \mathrm{e}^{\frac{t}{r}}\left(\frac{t}{r}\right)^{-n}\left[\Gamma(n+1)-\Gamma\left(n+1, \frac{t}{r}\right)\right]$

where $\Gamma(n), \Gamma(n, t)$ are the well-known gamma and incomplete gamma functions, respectively. Thus, we have the Sumudu transform of $Q(t)=t^{n}$ as

$$
\begin{aligned}
S\left[t^{n}\right] & =\left(r^{n}\left[\Gamma(n+1)-\Gamma\left(n+1, \frac{t}{r}\right)\right]\right)_{t=0}^{t=\infty} \\
& =r^{n}[(n !-0)-(n !-n !)]=r^{n} n !
\end{aligned}
$$

Case 2 Let $Q(t)=\frac{\sinh (a t)}{a}, 1>|a r|$. From (12), we obtain $f_{1}(t)$ as following

$$
f_{1}(t)=-\frac{\mathrm{e}^{a t}\left(2 a r \mathrm{e}^{\left(a+\frac{1}{r}\right) t}-a r+1-a r \mathrm{e}^{2 a t}-\mathrm{e}^{2 a t}\right)}{2 a\left(a^{2} r^{2}-1\right)}
$$

Considering the (2), (6), (14) and (32), we get the Sumudu transform of $Q(t)=\frac{\sinh (a t)}{a}$ 


$$
\begin{aligned}
S\left[\frac{\sinh (a t)}{a}\right]= & \left\{-\frac{r}{a^{2} r^{2}-1}+\frac{r \mathrm{e}^{-\left(a+\frac{1}{r}\right) t}}{2\left(a^{2} r^{2}-1\right)}-\frac{\mathrm{e}^{-\left(a+\frac{1}{r}\right) t}}{2 a\left(a^{2} r^{2}-1\right)}\right. \\
& \left.+\frac{r \mathrm{e}^{\left(a-\frac{1}{r}\right) t}}{2\left(a^{2} r^{2}-1\right)}+\frac{\mathrm{e}^{\left(a-\frac{1}{r}\right) t}}{2 a\left(a^{2} r^{2}-1\right)}\right\}_{t=0}^{t=\infty} \\
= & \frac{r}{1-r^{2} a^{2}}-\left\{\frac{r}{1-r^{2} a^{2}}-\frac{r}{2\left(1-r^{2} a^{2}\right)}+\frac{1}{2 a\left(1-r^{2} a^{2}\right)}\right. \\
& \left.-\frac{r}{2\left(1-r^{2} a^{2}\right)}-\frac{1}{2 a\left(1-r^{2} a^{2}\right)}\right\}=\frac{r}{1-r^{2} a^{2}}
\end{aligned}
$$

Case 3 Let $Q(t)=\mathrm{e}^{a t}, \frac{1}{r}>a$. We have the $f_{1}(t)$ as

$f_{1}(t)=\frac{\mathrm{e}^{\frac{t}{r}}-\mathrm{e}^{a t}}{1-a r}$

By substituting (34) into the right-hand side of (14), the Sumudu transform of $Q(t)=\mathrm{e}^{a t}$ is obtained as

$$
\begin{aligned}
S\left[\mathrm{e}^{a t}\right] & =\left\{\frac{1-\mathrm{e}^{\left(a-\frac{1}{r}\right) t}}{1-a r}\right\}_{t=0}^{t=\infty} \\
& =\frac{1-0}{1-a r}-\frac{1-1}{1-a r}=\frac{1}{1-a r}
\end{aligned}
$$

Case 4 Let $Q(t)=t \cos (a t)$. From (12), we have the $f_{1}(t)$ as

$$
\begin{aligned}
f_{1}(t)= & \frac{\left(r-a^{2} r^{3}\right) \mathrm{e}^{\frac{t}{r}}}{\left(r^{2} a^{2}+1\right)^{2}}-\frac{\left[t+a^{2} r^{2} t-r^{3} a^{2}+r\right] \cos (a t)}{\left(r^{2} a^{2}+1\right)^{2}} \\
& -\frac{\left[a r t+t a^{3} r^{3}+2 a r^{2}\right] \sin (a t)}{\left(r^{2} a^{2}+1\right)^{2}}
\end{aligned}
$$

and put (36) into right-hand side of (14), then we obtain the Sumudu transform of $Q(t)=t \cos (a t)$ as following

$$
\begin{aligned}
S[t \cos (a t)]= & \left\{\frac{r-a^{2} r^{3}}{\left(r^{2} a^{2}+1\right)^{2}}-\frac{\left[t+a^{2} r^{2} t-a^{2} r^{3}+r\right] \cos (a t)}{\mathrm{e}^{\frac{t}{r}}\left(r^{2} a^{2}+1\right)^{2}}\right. \\
& \left.-\frac{\left[2 a r^{2}+a^{3} r^{3} t+a r t\right] \sin (a t)}{\mathrm{e}^{\frac{t}{r}}\left(r^{2} a^{2}+1\right)^{2}}\right\}_{t=0}^{t=\infty} \\
= & \frac{r-a^{2} r^{3}}{\left(r^{2} a^{2}+1\right)^{2}}-0-0-\left(\frac{r-a^{2} r^{3}}{\left(r^{2} a^{2}+1\right)^{2}}-\frac{r-a^{2} r^{3}}{\left(r^{2} a^{2}+1\right)^{2}}\right)-0 \\
= & \frac{r-a^{2} r^{3}}{\left(r^{2} a^{2}+1\right)^{2}}
\end{aligned}
$$

Case 5 Let $Q(t)=\operatorname{erf}(t)$. By applying (2), (6), (12) and (14), we can easily write

$$
f_{1}(t)=\mathrm{e}^{\frac{t}{r}+\frac{1}{4 r^{2}}}\left[\operatorname{erf}\left(t+\frac{1}{2 r}\right)-\operatorname{erf}\left(\frac{1}{2 r}\right)\right]-\operatorname{erf}(t)
$$

Here $\operatorname{erf}(t)$ is a well-known error function. Thus, we obtain the Sumudu transform of $\operatorname{erf}(t)$ as

$$
\begin{aligned}
S[\operatorname{erf}(t)] & =\left\{\mathrm{e}^{\frac{1}{4 r^{2}}}\left[\operatorname{erf}\left(t+\frac{1}{2 r}\right)-\operatorname{erf}\left(\frac{1}{2 r}\right)\right]-\frac{\operatorname{erf}(t)}{\mathrm{e}^{\frac{t}{r}}}\right\}_{t=0}^{t=\infty} \\
& =\mathrm{e}^{\frac{1}{4 r^{2}}}\left[1-\operatorname{erf}\left(\frac{1}{2 r}\right)\right]-0-\left[\mathrm{e}^{\frac{1}{4 r^{2}}}\left(\operatorname{erf}\left(\frac{1}{2 r}\right)-\operatorname{erf}\left(\frac{1}{2 r}\right)\right)\right] \\
& =\mathrm{e}^{\frac{1}{4 r^{2}}}\left[1-\operatorname{erf}\left(\frac{1}{2 r}\right)\right]=\mathrm{e}^{\frac{1}{4 r^{2}}} \operatorname{erfc}\left(\frac{1}{2 r}\right)
\end{aligned}
$$

where $\operatorname{erfc}(t)$ is complementary error function.

Case 6 Let $Q(t)=\ln (t)$. Once again using the (12), we obtain $f_{1}(t)$ as

$f_{1}(t)=-\ln (t)-\mathrm{e}^{\frac{t}{r}} E_{1}\left(\frac{t}{r}\right)-\mathrm{e}^{\frac{t}{r}} \gamma-\mathrm{e}^{\frac{t}{r}} \ln \left(\frac{1}{r}\right)$

where $\gamma=0.5772156649 \ldots$ is the Eulers constant and $E_{1}(t)$ is a well-known exponential integral defined as in (27). If the same process is applied as in before, we obtain

$$
\begin{aligned}
S[\ln (t)] & =\left\{-\mathrm{e}^{-\frac{t}{r}} \ln (t)-E_{1}\left(\frac{t}{r}\right)-\gamma-\ln \left(\frac{1}{r}\right)\right\}_{t=0}^{t=\infty} \\
& =-0-0-\gamma-\ln \left(\frac{1}{r}\right)+\ln (t)-\gamma-\ln \left(\frac{t}{r}\right)+\gamma+\ln \left(\frac{1}{r}\right) \\
& =-\gamma+\ln (r)
\end{aligned}
$$

which is the Sumudu transform of $\ln (t)$.

\section{Conclusion}

We applied the variational iteration method for computing of the Laplace and Sumudu transforms of functions by using a different way. Results clearly show that unlike the literature and also classical computation, our presented method provides a powerful and easy calculation and also does not require too long operations. It is obviously indicated that the Laplace and Sumudu transforms of many functions, which are not mentioned here, can be computed by the VIM easily.

Open Access This article is distributed under the terms of the Creative Commons Attribution 4.0 International License (http://creativecommons.org/licenses/by/4.0/), which permits unrestricted use, distribution, and reproduction in any medium, provided you give appropriate credit to the original author(s) and the source, provide a link to the Creative Commons license, and indicate if changes were made.

\section{Appendix: Definition of Sumudu transform}

The famous integral transform which is used widely in Mathematics and the other disciplines is Laplace transform (see [11-13]). As the similar meaning, Watugala introduced a new integral transform for differential equations and engineering problems called Sumudu Transform [14-16] as following.

Let $T$ denotes the set of functions as

$$
T=\left\{f(t): \exists M, \tau_{1}, \tau_{2}>0,|f(t)|<M \mathrm{e}^{\frac{|t|}{\tau_{i}}}, \quad t \in(-1)^{i} \times[0, \infty)\right\}
$$


and Sumudu transform of functions $f(t)$ over the set of $T$ as

$F(u)=S[f(t): u]=\int_{0}^{\infty} f(u t) \mathrm{e}^{-t} \mathrm{~d} t, \quad u \in\left(-\tau_{1}, \tau_{2}\right)$

Also, a modified version of the definition of (43) is presented as

$F(u)=\int_{0}^{\infty} \frac{f(t) \mathrm{e}^{-\frac{t}{u}}}{u} \mathrm{~d} t, \quad u \in\left(-\tau_{1}, \tau_{2}\right)$

by Watugala [14-16], Belgacem [17, 18]. Furthermore, Sumudu transform is applied and improved for many areas of sciences (see some of [17-24]).

\section{References}

1. He, J.H.: Variational iteration method-a kind of non-linear analytical technique: some examples. Int. J. Nonlinear Mech. 34, 699-708 (1999)

2. He, J.H.: Some asymptotic methods for strongly nonlinear equations. Int. J. Mod. Phys. B 20(10), 1141-1199 (2006)

3. He, J.H.: Variational iteration method-some recent results and new interpretations. J. Comput. Appl. Math. 207(1), 3-17 (2007)

4. Xu, L., Lee Eric, W.M.: Variational iteration method for the magnetohydrodynamic flow over a nonlinear stretching sheet. Abstr. Appl. Anal. (2013). https://doi.org/10.1155/2013/573782

5. Wazwaz, A.M.: The variational iteration method for solving linear and nonlinear ODEs and scientific models with variable coefficients. Central Eur. J. Eng. 4(1), 64-71 (2014)

6. Matinfar, M., Ghasemi, M.: Application of variational iteration method to nonlinear heat transfer equations using He's polynomials. Int. J. Numer. Methods Heat Fluid Flow 23(3), 520-531 (2013)

7. Malvandi, A., Ganji, D.D.: A general mathematical expression of amperometric enzyme kinetics using He's variational iteration method with Pade approximation. J. Electroanal. Chem. 711, 32-37 (2013)

8. Fatoorehchi, H., Abolghasemi, H., Magesh, N.: The differential transform method as a new computational tool for Laplace transforms. Natl. Acad. Sci. Lett. 38(2), 157-160 (2015)

9. Abbasbandy, S.: Applications of He's homotopy perturbation method for Laplace transform. Chaos Solitons Fractals 30, 12061212 (2006)
10. Babolian, E., Biazar, J., Vahidi, A.R.: A new computational method for Laplace transforms by decomposition method. Appl. Math. Comput. 150, 841-846 (2004)

11. Doetsch, G.: Introduction to the Theory and Application of the Laplace Transformation. Springer, Berlin (1974)

12. Williams, J.: Laplace Transforms, Problem Solvers. George Allen and Unwin, Crows Nest (1973)

13. Widder, D.V.: The Laplace Transform. Princeton Mathematical Series. Princeton University Press, Princeton (1941)

14. Watugala, G.K.: Sumudu transform: a new integral transform to solve differential equations and control engineering problems. Int. J. Math. Educ. Sci. Technol. 24(1), 35-43 (1993)

15. Watugala, G.K.: Sumudu transform new integral transform to solve differential equations and control engineering problems. Math. Eng. Ind. 6(4), 319-329 (1998)

16. Watugala, G.K.: The Sumudu transform for functions of two variables. Math. Eng. Ind. 8(4), 293-302 (2002)

17. Belgacem, F.B.M., Karaballi, A.A.: Sumudu transform fundamental properties investigations and applications. J. Appl. Math. Stoch. Anal. (2006). https://doi.org/10.1155/JAMSA/2006/91083

18. Belgacem, F.B.M., Karaballi, A.A., Kalla, S.L.: Analytical investigations of the Sumudu transform and applications to integral production equations. Math. Probl. Eng. 3, 103-118 (2003)

19. Belgacem, F.B.M.: Sumudu transform applications to Bessel functions and equations. Appl. Math. Sci. 4(74), 3665-3686 (2010)

20. Weerakoon, S.: Application of Sumudu transform to partial differential equations. Int. J. Math. Educ. Sci. Technol. 25(2), 277-283 (1994)

21. Kilicman, A., Gadain, H.E.: An application of double Laplace transform and double Sumudu transform. Lobachevskii J. Math. 30(3), 214-223 (2009)

22. Abdul, Rahman N.A., Ahmad, M.Z.: Applications of the fuzzy Sumudu transform for the solution of first order fuzzy differential equations. Entropy 17, 4582-4601 (2015)

23. Kilicman, A., Gadain, H.E.: On the applications of Laplace and Sumudu transforms. J. Frankl. Inst. 347, 848-862 (2010)

24. Kilicman, A., Eltayeb, H., Agarwal, R.P.: On Sumudu transform and system of differential equations. Abstr. Appl. Anal. (2010). https://doi.org/10.1155/2010/598702

Publisher's Note Springer Nature remains neutral with regard to jurisdictional claims in published maps and institutional affiliations 